\title{
Potential-Dependent Changes of the Surface Tension of Water
}

\author{
Yuri Pivovarenko \\ Research and Training Center 'Physical and Chemical Materials Science' Under Kyiv Taras Shevchenko University and NAS of Ukraine, \\ Kiev, Ukraine
}

\section{Email address:}

y.pivovarenko@gmail.com

\section{To cite this article:}

Yuri Pivovarenko. Potential-Dependent Changes of the Surface Tension of Water. Fluid Mechanics. Vol. 3, No. 4, 2017 , pp. $29-32$. doi: 10.11648/j.fm.20170304.11

Received: May 11, 2017; Accepted: May 31, 2017; Published: June 30, 2017

\begin{abstract}
It was shown previously that the shape of the crystals formed during the evaporation of salt solutions depends on the sign of the electric potential of such solutions. It has been particularly found that the evaporation of salt solutions with positive electric potential is accompanied by the formation of cubic crystals and the evaporation of salt solutions with negative electric potential is accompanied by the formation of needle-like crystals. The obtained results stimulated further studies of the properties of water and aqueous solutions, which depend on the electric potential. During these studies it was found that the surface tension of the water clearly depends on its electrical potential: an increase in the positive electric potential of water is accompanied by an increase in its surface tension and increase the negative electrical potential of the water is accompanied by a decrease of its surface tension. It was also discovered that the electrical potential of the water determines its ability to hydrate the polymers of biological origin. It is shown that water with a positive potential is better hydrates biological polymers than water with negative potential.
\end{abstract}

Keywords: Electrical Potential, Water, Surface Tension, Muscle Contraction

\section{Introduction}

Polymorphism of the salt crystals is the basis of the known method of medical diagnosis, is known as a method of arborization [1-4]. It has been shown that the shape of the crystals resulting from evaporation of salt solutions depends on the sign of the electric potential of the solution. It was particularly shown that the evaporation of saline solutions with a positive electric potential is accompanied by formation of cubic crystals, and evaporation of salt solutions with negative electric potential is accompanied by formation of needle-like crystals (Figure 1) [5].

Despite the detected correlation, the nature of the forces causing the polymorphism of the salt crystals remained unclear to us. We therefore continued research in this area. In other words, we continued to explore the properties of water and aqueous solutions, which depend on their electrical potential (charge). Working in this direction we found that the surface tension of water depends on its electric potential in a certain way: positive electrification of water causes an increase in its surface tension but the negative electrification of water causes a decrease in its surface tension.

We also found that the electric potential of the water determines its ability to hydrate the polymers of biological origin. It was in addition shown that water with a positive potential is better hydrates biological polymers than water with negative potential. In our opinion, these relationships enable us to better understand the physical nature of the phenomena of muscle contraction. Here we offer results on the basis of which we make such conclusions.

\section{Material and Methods}

First, we believe it is necessary to define the terminology that we use in this article. The term "uncharged water" we conventionally understand water, which was used as a control: we assumed that the electric potential of this water is $0 \mathrm{mV}$. Uncharged water was obtained during storage of distilled water in a closed aluminum containers: It is considered that in such circumstances, the electric charges of 
water are concentrated on the outer surface of the container [6].

Water with a positive electric potential we have obtained in two ways:

(a) By passing through uncharged water of gaseous oxygen.

(b) By filtration of uncharged water through the silica gel.

It is known that when in contact with water, oxygen gas exhibits the properties of a sorbent of aqueous electrons, and the silica gel exhibits the properties of a sorbent of aqueous hydroxyl ions [7].

Water with a negative electric potential we have also obtained in two ways:

(a) By passing through uncharged water of gaseous hydrogen.

(b) By filtration of uncharged water through the activated carbon.

It is known that when in contact with water, hydrogen gas is the electron donor, and activated carbon exhibits the properties of a sorbent of aqueous hydrogen ions [7].

We obtained water with the desired value of the electric potential in two ways:

(a) By varying the depth of the layer of sorbent through which filtered water is discharged.

(b) Varying the time during which the gas passed through the uncharged water.

The electric potential of the electrically charged water, we measured relative to the uncharged water, the potential of which we have conventionally taken to be $0 \mathrm{mV}$. In fact, the electric potential of the charged water, we measured the potential flow or potential filtering [8].

\section{Results and Discussion}

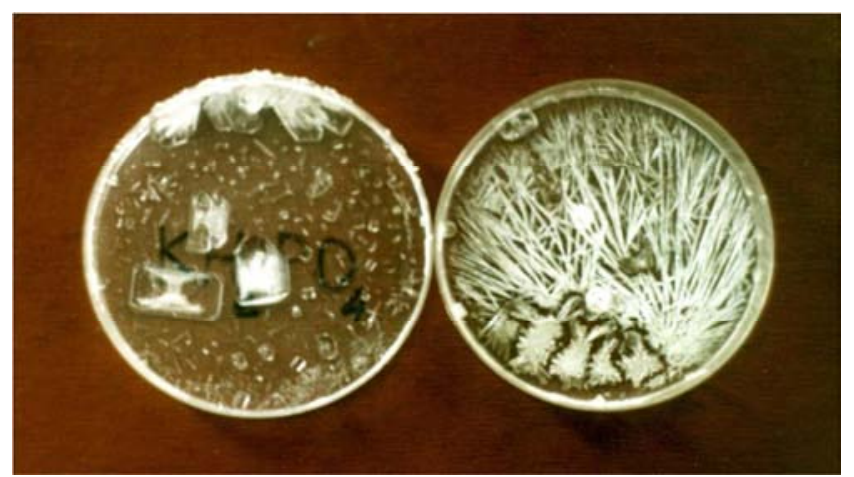

Figure 1. This is the crystals of $\mathrm{KH}_{2} \mathrm{PO}_{4}$. Left: the rhombic crystals formed upon drying of an aqueous solution of $\mathrm{KH}_{2} \mathrm{PO}_{4}$ prepared on the water with positive electric potential. Right: the needle-shaped crystals formed upon drying of an aqueous solution of $\mathrm{KH}_{2} \mathrm{PO}_{4}$ prepared on the water with negative electric potential [5].

In our previous study, we used the water with a different electric potential [5]. When conducting these experiments, we drew attention to the fact that the surface tension of water depends on its electrical potential. This relationship was the fact that the surface tension of water with a positive potential greater than the surface tension of water with negative potential. Here we propose to verify the existence of such dependencies through simple experiments.

So, if to pour $5 \mathrm{ml}$ of water (exactly!) with a negative potential in a standard Petri dish and mix, you can see that the water completely covers the bottom of a Petri dish (Figure 2, left).

On the other hand, if to pour $5 \mathrm{ml}$ of water (exactly!) with a positive potential in a standard Petri dish and mix, you can see that such water will not cover all the bottom of a Petri dish (Figure 2, right).

In our view, such a distinction clearly demonstrates that the forces acting on the surface of the water depends on its electric potential. We explained this by the fact that water with a positive potential has a greater surface tension than water with negative potential.

(Apparently, this distinction should be defined more precisely. The forces acting between molecules that reside on the surface of positively charged water are stronger than the forces acting between molecules that reside on the surface of negatively charged water. On the other hand, the interaction between the negatively charged molecules of water and the surface of the glass are stronger than the same interaction between the positively charged water and glass surface.)

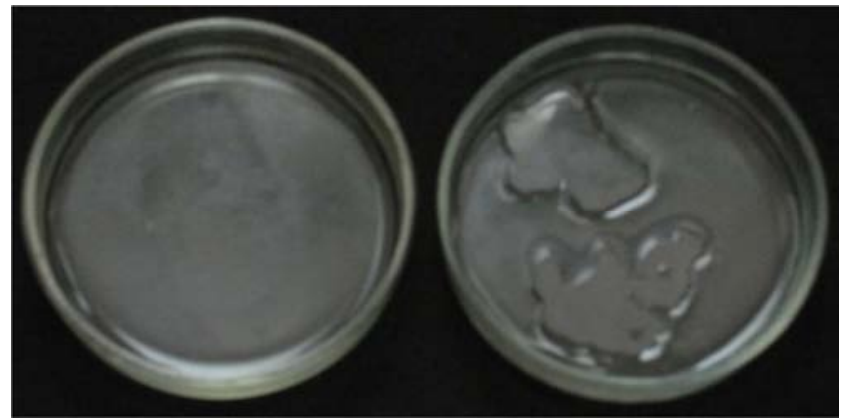

Figure 2. Left: $5 \mathrm{ml}$ of water with an electric potential of -200 $\mathrm{mV}$ cover all the bottom of a Petri dish. Right: $5 \mathrm{ml}$ of water with an electric potential of $+200 \mathrm{mV}$ do not cover the bottom of a Petri dish; the surface of such water decreases rapidly after mixing.

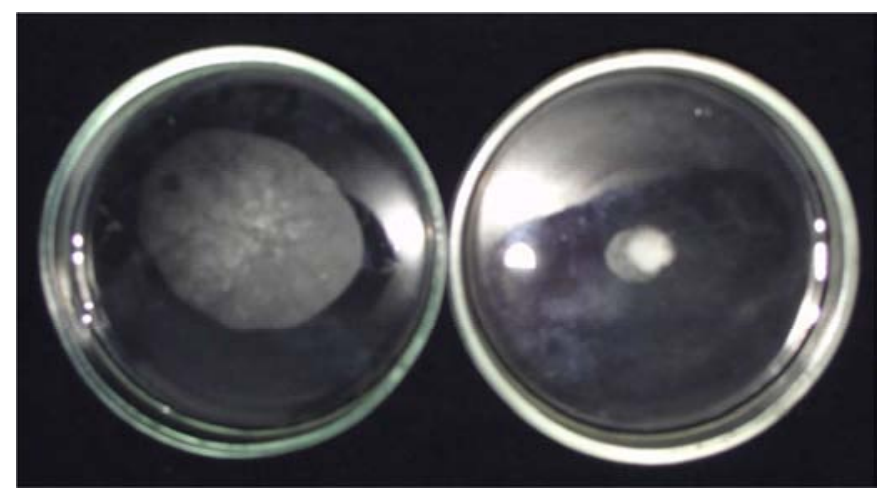

Figure 3. Left: the starch powder covers the surface of the water with potential $+250 \mathrm{mV}$ practically wholly Right: powder starch remains in the same place where it was put in water potential $-200 \mathrm{mV}$.

The detected difference can be confirmed and visualized using simple experiments. For example, the difference in surface tension can be easily visualized by means of a powder of starch deposited on the surface of charged water. 
By this simple test, you can see that the forces acting on the surface of positively charged water dispense powder starch on the surface of the water (Figure 3, left); such a distribution of powder of starch takes place over $1-2$ seconds.

It can also be seen that the surface forces of negatively charged water do not distribute the starch powder across the water surface (Figure 3, right). Moreover it can be observed that the starch powder sinks into negatively charged water. In our opinion, this experiment clearly demonstrates the difference in the surface tension of water with positive and negative potentials.

Not less convincing is the result of the following experiment in which we also used powder starch. For this experiment, we used two vessels. The first vessel was filled with water with a potential of $+500 \mathrm{mV}\left(\sim 20^{\circ} \mathrm{C}\right)$, and the second vessel was filled with water with a potential of -500 $\mathrm{mV}\left(\sim 20^{\circ} \mathrm{C}\right)$. After that, we introduced and stirred in both vessels of $100 \mathrm{mg}$ of starch powder. Then we put both vessels in a place that was removed from heat sources and protected from direct sunlight. After $30 \mathrm{~min}$. it was seen that the starch did not swell in a bottle containing water with negative potential (Fig. 4, left), but swelled in a bottle filled with water with a positive potential (Fig. 4, right).

The result of the last experiment, we also explained that the surface tension of water with a positive potential is much greater than the surface tension of water with negative potential. In any case, this result shows that the positively charged water is able to quickly hydrate the starch (Figure 4, right).

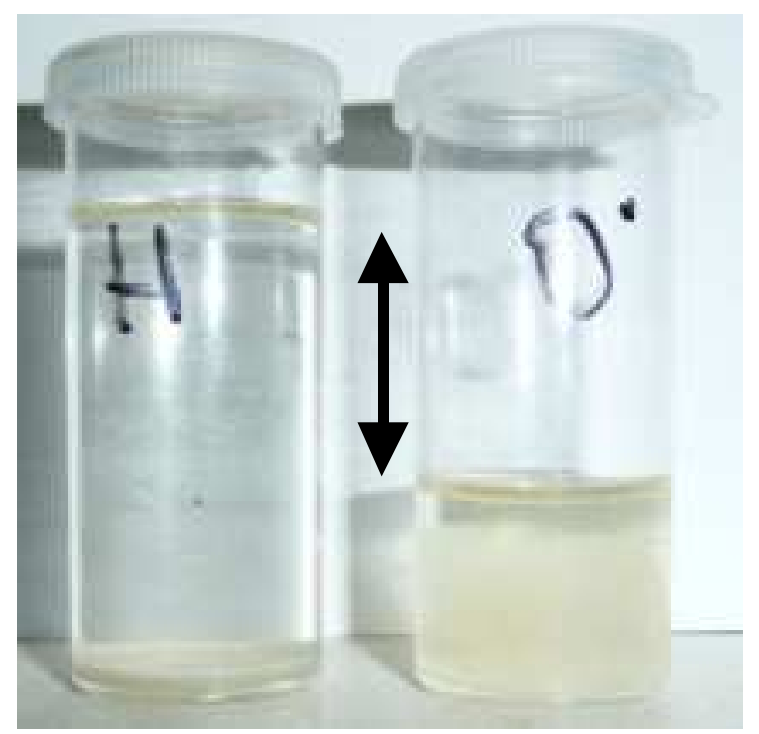

Figure 4. There is a swelling of starch in water with a different electric potential. Starch does not swell in water with the potential of-500 (left) and swells in water with a potential of $+500 \mathrm{mV}$ (right).

Water with negative potential was obtained by bubbling hydrogen (left); water with a positive potential was obtained by bubbling oxygen (right).

Water with a positive potential can evaporate even from a closed plastic dishes: the arrow shows how during the day decreased the water level to the positive potential.

When conducting the last experiment, we observed another interesting property of water with positive potential. As you can see (Fig. 4, right), this water is able to penetrate through the plastic and evaporate from a closed polyethylene bottle. It should be noted that the increase in the permeability of polyethylene to water vapor is known: this phenomenon was observed earlier in the oxidation of polyethylene ROS induced by ionizing radiation [9]. Recently we proposed a detailed analysis of the physical forces causing this phenomenon [10].

It should be noted that here we showed different surface tension electrically charged water exclusively with starch. However, similar results can be obtained using other polymers of biological origin. Very impressive looking changes of the fibers of the sodium salt of DNA deposited on the surface of the positively charged water. So, we watched as the fiber of the sodium salt of DNA form a transparent film on the surface of such water; this transformation occurs within $1-2$ seconds [11].

We hope that the results of our experiments (Figure 2-4) convinced you that the surface tension of water depends on its electric potential. We offer the following formulation of this dependence: the surface tension of water having a positive electrical potential is always higher than the surface tension of water having a negative electrical potential. It is also possible to formulate: the increase in the electric potential of the water increases its surface tension, and the reduction of the electric potential of water causes a decrease of its surface tension. The latter reflects the results of our experiments which showed that changing the electric potential of water can be directed to change its surface tension. (Unfortunately, we are unable to visualize it.) In other words, changing the electrical potential of the water, you can control its surface tension.

It should be noted that this visual similarity has a profound basis, since a similar dependence of surface tension on the electric potential is known also for other liquids. For example, the existence of such dependence is shown for liquid mercury when studying the physical nature of the phenomenon, known as the "mercury heart beating" $[12,13]$. Special attention deserves the work, the authors showed that the surface tension of mercury, having a positive electrical potential greater than the surface tension of mercury, having a negative electric potential [12].

Also in favor of the generality of this dependence of surface tension on the electric potential of the study results show the micro hardness of monocrystalline silicon. So, it was clearly established that the negative electrification of monocrystalline silicon is accompanied by a $20 \%$ decrease in hardness of its surface layer. It was also found that the subsequent positive charging leads to the restoration of micro-hardness of silicon single crystals [14].

In our opinion, this dependence is very important for understanding the nature of the physical phenomena that underlie muscle activity. We assume two phases of this activity. In the first stage, the surface tension of muscle fibers is increased under the action of positive potential. At the same time, the muscles tense due to the increase in the extent 
of hydration of the internal components, as well as actomyosin complex [15]. In the second stage, the surface tension of muscle fibers decreases under the action of negative potential. At the same time, the muscles relax due to the decrease of the degree of hydration of the internal components, as well as actomyosin complex. (It should be noted that none of the known authors not bound by state actomyosin complexes and their degree of hydration.)

The proposed stage can be presented, focusing on the shape of the crystals formed by evaporation of saline solutions made with water having different electric potential: the formation of cubic crystals from solutions with a positive electric potential reflects the stage of muscle tension and the formation of needle-like crystals from solutions with negative electric potential reflects the stage of muscle relaxation (Figure 1).

\section{Conclusion}

The surface tension of water depends on its electric potential (charge). This dependence can be formulated as follows: the surface tension of water having a positive electrical potential is always higher than the surface tension of water having a negative electrical potential. This dependence can also be formulated differently: the increase in the electric potential of the water increases its surface tension, and the reduction of the electric potential of water causes a decrease of its surface tension.

\section{References}

[1] Vasilchenko G. S. (1990) Sexual pathology. Moscow: Medicine.

[2] Braga D., Grepioni F., Maini L., and Polito M. (2009) Crystal polymorphism and multiple crystal forms. Struct. Bond., 132, 25-50.

[3] Yakhno T. A. (2011) Complex pattern formation in sessile droplets of protein-salt solutions with low protein content. What substance fabricates these patterns? Physical Chemistry, $1,10-13$.

[4] Markevich V. E., Kirilenko E. A., Petrashenko V. A., Zabolotskaja T. Y., and Bilokon M. A. (2014) Methods of wedge dehydration of biological fluids. Morphologia, 8 (1), 113-117.
[5] Pivovarenko Yu. V. (2016) Nature of the polymorphism of salt crystals in the aspect of arborization diagnostic method. Morphologia, 10 (1), 72-6.

[6] Purcell E. (1971) Electricity and magnetism. BPC, 2. Moscow: Nauka.

[7] Nekrasov B. V. (1974) Bases general chemistry, 1. Moscow: Chemistry.

[8] Fridrichsberg D. A. (1974) Course of colloid chemistry. Leningrad: Chemistry.

[9] Spangenberg J. E., and Vennemann T. W. (2008) The stable hydrogen and oxygen isotope variation of water stored in polyethylene terephthalate (PET) bottles. Rapid Commun. Mass Spectrom., 22, 672-676.

[10] Pivovarenko, Y. (2015) A Charge Distribution in the Earth's Atmosphere. American Journal of Physics and Applications. 3 (3), 67-68.

[11] Veselsky S., Pivovarenko, Y., and Lyakhov A. (2006) Properties of nucleic acids in aqueous solutions with different electric potential. Bulletin of Kiev. University. Ser.: Problems of physiological functions regulation, 1, 14-17.

[12] Najdoski M., Mirceski V., Petruševski V. M., and Demiri Sani. (2007) Mercury beating heart: Modifications to the classical demonstration. J. Chem. Educ., 84 (8), 1292-1295.

[13] Shu-Wai Lin, Keizer J., Rock P. A., and Stenschke H. (1974) On the mechanism of oscillation in the "Beating Mercury Heart". PNAS, 71 (11), 4477-4481.

[14] Steblenko L. P., Naumenko S. M., Pivovarenko Y. V., Vesna G. V., Kuryluk A. M., Kalinichenko D. V., and Volkova, T. V. (2012) The effect of electrochemical activation of water on the micro-hardness of crystalline silicon. Works of the 3rd International Conference "Modern problems of condensed matter physics", 10 - 13 October 2012, Kyiv, Ukraine, 113114.

[15] Andreev O. A., and Reshetnyak Y. K. (2007) Mechanism of formation of actomyosin interface. JMB, 365 (3), 551-554.

[16] Whittaker E. T. (1951) A history of the theories of aether and electricity, 1. London: Nelson.

[17] Kamen G. (2004) Electromyographic Kinesiology. In Robertson D. G. E. et al. Research Methods in Biomechanics. Champaign, IL: Human Kinetics Publ.

[18] Roe S. M., Johnson C. D., and Tansey E. A. (2014) Investigation of physiological properties of nerves and muscles using electromyography. Adv. Physiol. Educ., 38, 348-354. 